\title{
A Computational Framework to Benchmark Basket Catheter Guided Ablation
}

\author{
Martino Alessandrini ${ }^{1}$, Maddalena Valinoti $^{1}$, Axel Loewe ${ }^{2}$, Tobias Oesterlein ${ }^{2}$, Olaf Dössel ${ }^{2}$, \\ Cristiana Corsi ${ }^{1}$, Stefano Severi ${ }^{1}$ \\ ${ }^{1}$ University of Bologna, Cesena, Italy \\ ${ }^{2}$ Institute of Biomedical Engineering, Karlsruhe Institute of Technology (KIT), Karlsruhe, Germany
}

\begin{abstract}
Atrial fibrillation (AF) ablation guided by basket catheter mapping has shown to be beneficial. Yet, the initial excitement is mitigated by a growing skepticism due to the difficulty in verifying the protocol in multicenter studies. Overall, the underlying assumptions of rotor ablation require further verification. The aim of this study was therefore to test such hypotheses by using computational modeling.

The $3 D$ left atrial geometry of an AF patient was segmented from a pre-operative MR scan. Atrial activation was simulated on the $3 D$ anatomy using the monodomain approach and a variant of the Courtemanche action potential model. Ablated tissue was assigned zero conductivity. Reentry was successfully initialized by applying a single suitably delayed extra stimulus. Unipolar electrograms were computed at the simulated electrode positions. The final dataset was generated by varying location of reentry and catheter position within the LA. The effect of inter-electrode distance and distance to the atrial wall was studied in relation to the ability to recover rotor trajectory, as computed by a novel algorithm described here. The effect of rotor ablation was also assessed.
\end{abstract}

\section{Introduction}

Pulmonary vein (PV) ablation is typically employed to treat atrial fibrillation (AF). Yet, further substrate ablation is often necessary. Hereto, Focal Impulse and Rotor Modulation (FIRM) guided ablation has raised great interest given the high reported success rates. Yet, the initial excitement was mitigated by a growing skepticism due to the difficulty in verifying the protocol in multicenter studies. The underlying assumptions of the FIRM protocol still need verification: i) AF is sustained by stable rotors; ii) a rotor can be terminated by ablating its core; iii) basket catheters (such as Constellation, Boston Scientific) allow reliable rotor mapping.

Here, we use computational modelling to test some of the underlying assumptions and benchmark basket-guided ablation. In particular, we tested i) if the rotor core can be reliably localized by a grid of electrodes and how the number/resolution of electrodes and the distance from the atrial wall can affect such localization ii) if simulated ablation is able per se to terminate $\mathrm{AF}$ and if the spatial information derived from the EGMs is critical for the procedure success.

\section{Materials and Methods}

\subsection{Clinical Data}

The 3D left atrial geometry was segmented from a preoperative MR scan performed on a paroxysmal AF patient selected for AF ablation at the Bufalini Hospital in Cesena, Italy.

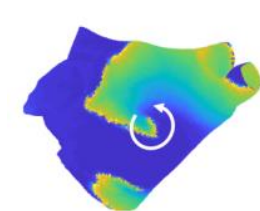

(a) posterior wal

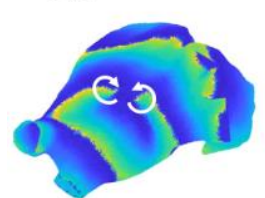

(c) roof

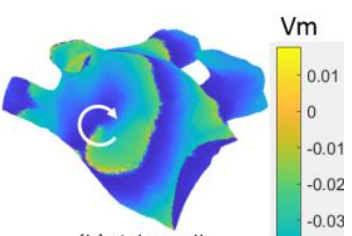

(b) right wall

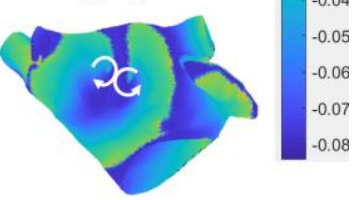

(d) posterior wall
Figure 1: Representative screenshots of the 4 reentry simulations induced by ectopic stimuli from different locations. Color encodes trans membrane potential (V).

\subsection{Modeling of Activation}

Atrial activation was simulated on the 3D anatomy using the monodomain approach [1] and a variant of the Courtemanche et al. action potential model accounting for spatial heterogeneity and AF related ionic remodeling [2]. Sinus activation was simulated by pacing the model at the insertion point of the Bachmann bundle and at the Fossa Ovalis with a physiological delay of $26 \mathrm{~ms}$. Re-entry was initiated by placing an extra stimulus in a circular area of 
tissue of radius $10 \mathrm{~mm}$. We generated 4 different simulations by applying the extra stimulus in different locations: posterior wall; right lateral wall below the inferior PV; LA roof and posterior wall towards the roof. In all cases, a "figure of eight" pattern was obtained. The distance between the 2 circuits varied depending on the location (cf. Fig. 1). Two seconds of activation were simulated. Sinus activation was applied once and then suspended.

\subsection{Intra Atria Pseudo Electrograms}

Unipolar electrograms (EGMs) were simulated by using the assumption of an infinite homogeneous medium [3]. Sampling frequency was $1000 \mathrm{~Hz}$.

To study the effect of sampling density and distance to the atrial wall, we generated a set of "toy" catheters composed of a 2D grid of point electrodes. One with 9x9 electrodes with a relative distance of $3 \mathrm{~mm}$ and one with $5 \times 5$ electrodes and $6 \mathrm{~mm}$. The patch size was therefore

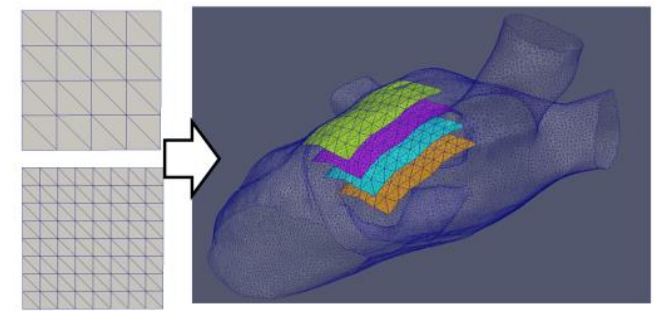

Figure 2: Figure 2: Grid catheter placement. The grid is first computed in a 2D coordinate system and then adapted to the 3D atrial geometry. The different colors represent grids at different distance from the wall: green (contact); magenta $(0.5 \mathrm{~cm})$; cyan $(1 \mathrm{~cm})$; orange $(1.5 \mathrm{~cm})$.

$24 \times 24 \mathrm{~mm}$ in both setups. The grid was first placed in contact to the atrial wall and centered at the location of the reentry. The catheter was then moved away from the atrial wall inside the cavity in steps of $5 \mathrm{~mm}$ up to a maximum distance of $15 \mathrm{~mm}$ (cf. Fig. 2). To test the ability to distinguish two simultaneous reentries, we chose to cover both reentries in the figure of eight in 2 cases ((c) and (d) in Fig. 1).

As such, we generated a dataset of 32 simulations: 4 rotor positions times two values of inter-electrode spacing ( 3 and $6 \mathrm{~mm}$ ) times 4 values of distance to the wall (contact, 5, 10 and $15 \mathrm{~mm}$ ).

\subsection{Rotor Tracking}

The algorithm for rotor tracking is described more extensively in [5]. Briefly, rotor detection is based on the concept of phase singularities (PS). Hereto, the simulated EGM signals were first converted into phase signals by using a modified version of the "sinusoidal recomposition" approach. The extracted phase values where then mapped from the electrode positions to the $3 \mathrm{D}$ geometry by nearest neighbor interpolation with a cut-off value of $13 \mathrm{~mm}$ (cf. Fig. 3). The algorithm then proceeds by looking for closed loops with monotonically increasing/decreasing phase values and a jump of at least $2 \pi$ as per definition of a "phase singularity". The search is progressively extended to neighboring regions, i.e. sharing an interface in the 3D reconstruction (Fig.3(b)).

The PS is classified as a rotor if its lifespan is greater than one AF cycle length, computed by considering the

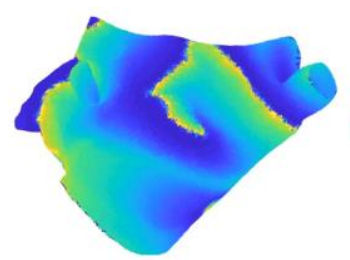

(a) Simulated activation map

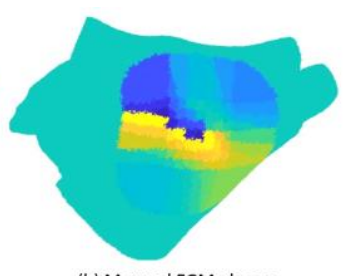

(b) Mapped EGM phases
Figure 3: Transmembrane voltage distribution (a) and mapped acquisition (phase) from the grid catheter (in contact with $3 \mathrm{~mm}$ inter-electrode spacing).

inverse of the dominant frequency (DF) (DF $\sim 7.8 \mathrm{~Hz}$ in our simulations). In addition the persistence in time of the rotor was estimated considering a threshold on the maximum distance between the coordinates of its phase singularity points estimated frame by frame. The threshold was fixed at $0.8 \mathrm{~cm}$ for the $9 \times 9$ grid and $1.7 \mathrm{~cm}$ for the $5 \times 5$ grid.

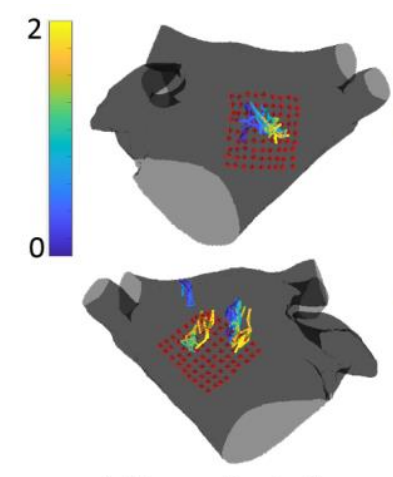

(a) traced trajectory

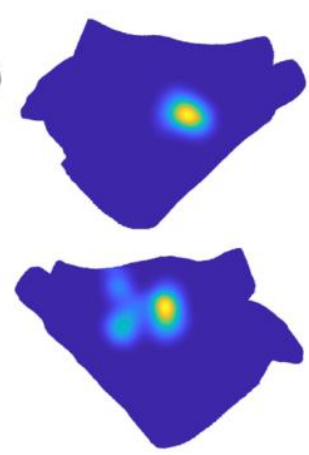

(a) density map
Figure 4: Traced "ground truth" PS trajectory (a) and associated density maps (b) for the cases (a) and (d) of Fig. 1. Trajectory color in (a) encodes time in seconds. The position of the sensing grid is denoted by the red asterisks (contact catheters with $3 \mathrm{~mm}$ spacing).

\subsection{Simulated Ablation}

Ablation was simulated by assigning zero conductivity to the ablated tissue. Hereto, we evaluated 2 scenarios in 

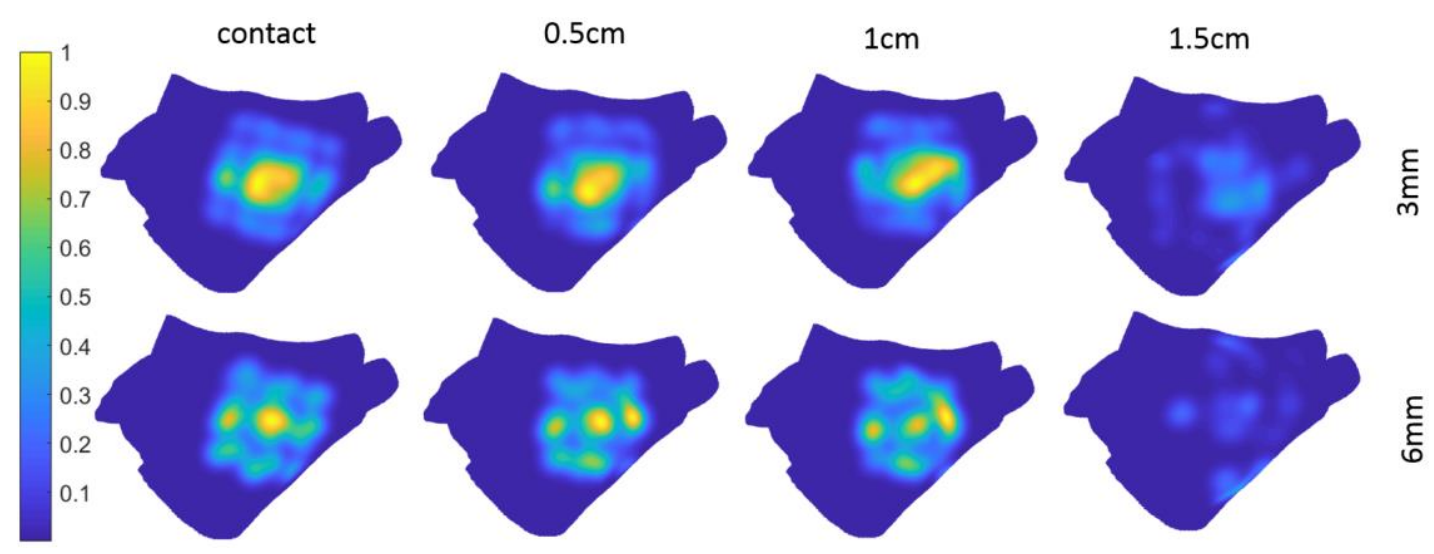

Fig. 5: Combined effect of grid spacing (rows) and wall distance (columns) on the reconstructed phase singularity density maps.

which the ablated region was either in the shape of a circle or a cross. Circle diameter and cross arms were both equal to $7.5 \mathrm{~mm}$. Shape and size were chosen consistently with [4]. The ablation was centered based on the indication of the rotor tracking algorithm. All lesions were fully transmural.

\subsection{Experimental Evaluation}

The output of the rotor tracking algorithm was compared against the "ground-truth" trajectory, detected manually on the full resolution activation map derived directly from the transmembrane voltage distribution. The results are presented as density maps. Hereto, all points (either on the ground truth trajectory, the estimated one or corresponding to PS) were collected in a point cloud $\boldsymbol{y}=$ $\left\{\mathbf{y}_{\mathbf{i}}\right\}$. The density $d_{\boldsymbol{i}}$ of a mesh node $\mathbf{x}_{\boldsymbol{i}}$ was computed as $d_{i}=\sum_{j} f\left(|| \mathbf{x}_{i}-\mathbf{y}_{\mathrm{j}}||\right), \quad$ with $\quad f(z)=\exp \left(-z^{2} /\left(2 \sigma^{2}\right)\right)$ and $\sigma=3 \mathrm{~mm}$ (Fig. 4).
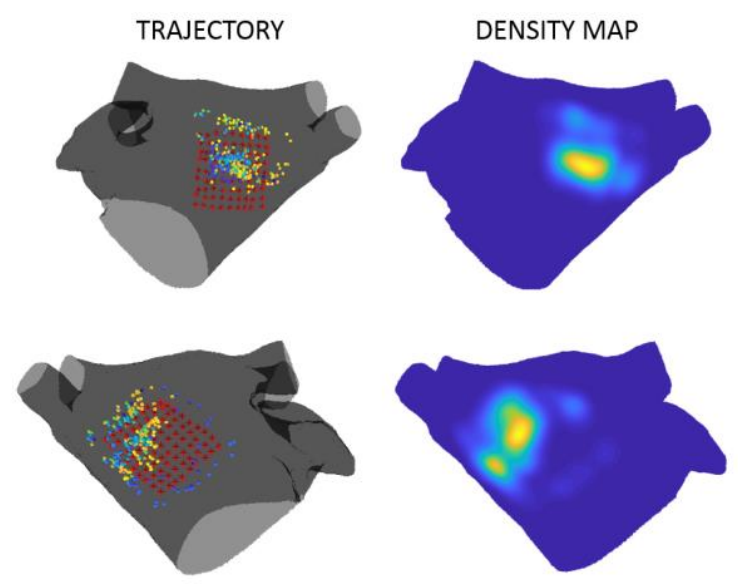

Fig. 6: Estimated PS trajectory (left) based on simulated EGMs and associated density map (right) for the same simulations as in Fig. 4.

\section{Results}

The effect of distance to the atrial wall and interelectrode distance is illustrated in Fig. 5 for the PS computed from the simulated EGMs. By comparison with the "ground truth" (first row of Fig. 4) one can notice a large number of "spurious" phase singularities, i.e. not associated to a rotation center. In general, as intuitively expected, an increase in distance implies a smoothing of the phase density maps while and an increase in spacing gives rise to a larger number of localized maxima. Moreover, a wall distance of $1.5 \mathrm{~mm}$ is obviously too large for a reliable phase computation. In this setting (cf. Fig. 2) the catheter is almost midway between the wall of interest and the anterior wall and it is therefore highly influenced by the field from the opposite (anterior) wall.

Fig. 6 shows the density map after spurious phase singularities have been cleaned by the rotor classification algorithm for the same simulations as in Fig. 4 in the case of a contact catheter with $3 \mathrm{~mm}$ spacing. In the first simulation (top row), there was a single rotor well centered in the acquired region, and the algorithm could reconstruct its position with very good accuracy $(1.2 \mathrm{~mm}$ distance between the maxima). The second case (bottom row) was more complex, with two rotors meandering on the region covered by the catheter (cf. Fig. 4 bottom row): one more marginal (on the right) which persisted throughout the simulation while a second one, more central to the catheter, appeared at $1.1 \mathrm{~s}$. In this case, the algorithm identified one single peak corresponding to the well-covered rotor $(0.7 \mathrm{~mm}$ distance between the maxima) while the more marginal one remained undetected.

A preliminary evaluation of ablation was performed on the simulation of Fig, 7. The result is summarized in Fig.5. In the simulation without ablation, there are two dominant rotors: one in the posterior wall (the ablated one) and one in the lateral wall (gray arrow in top left panel). Due to ablation with a cross shaped lesion (mid 
row) the ablated rotor initially shifts from its original position towards the roof $(\mathrm{t}=3.2 \mathrm{~s})$. After few seconds $(\mathrm{t}=$ $6.7 \mathrm{~s}$ ) an anatomical reentry appears anchored by the right superior PV (RSPV). Eventually the two reentries (2 and 3) merge into a single one rotating around the RSPV. Ablation with a circle region generates a more complex pattern. Initially, the ablated rotor is shifted to the roof, then two anatomical reentries appear around the LSPV, first, and around the RSPV.

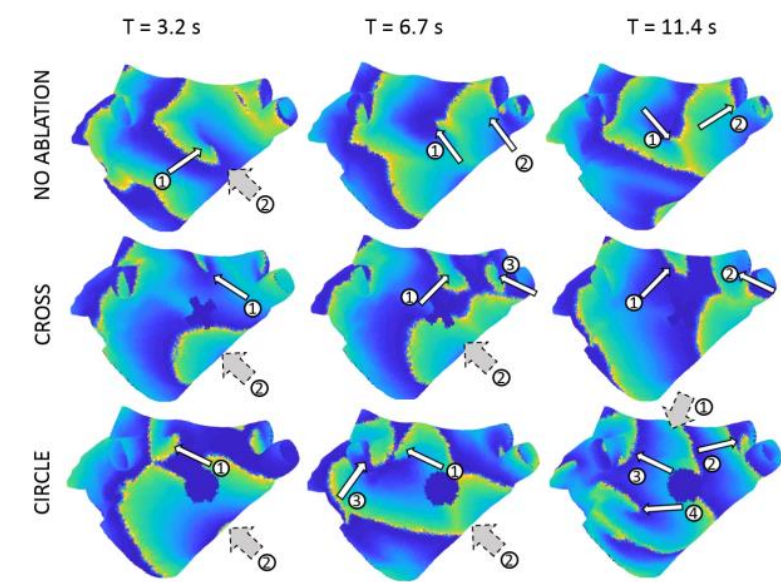

Fig. 7: Simulated ablation. Extracted video frames of the simulations without ablation (top row) and with ablation with cross (middle row) and circular lesions (bottom row) at corresponding time instants. The arrows identify the dominant reentrant circuits (see main text).

\section{Discussion and Conclusion}

Due to the sparse sampling, PS alone was not a reliable indicator of a center of reentry. Yet, since PS tended to accumulate around the center of rotation while spurious PS were more uniformly distributed, an integral "phasedensity" map were found useful to provide a rough indication of a rotor center. Such maps became highly multimodal and therefore unreliable for a grid spacing of $6 \mathrm{~mm}$. An increase in distance had 2 effects, i) a loss in spatial resolution, ii) an increased sensitivity to "far-field" effects (i.e. coming from regions other than the mapped one). The way these two factors interplay is strictly case specific. In the case of Fig. 5, a distance up to $1 \mathrm{~cm}$ was still appropriate to visualize the rotor center.
The employed rotor tracking algorithm included a rotor classification algorithm to get rid of spurious PS. The algorithm was tested on grids at contact with a $3 \mathrm{~mm}$ spacing and was found to provide accurate estimates of a rotor center. Yet, the estimate is reliable only on regions well centered with the catheter while boundary regions suffer from border artefacts.

Catheter driven ablation had the effect of detaching the catheter from its rotating core but it did not stop the fibrillation. Rather, he fibrillation pattern became in general more complex and less organized, in particular for a circular lesion.

This adds up to previous modelling studies pointing to the potential pitfalls of multi-electrode catheters for $\mathrm{AF}$ mapping [6].

\section{Acknowledgements}

This project has received funding from the European Union's Horizon 2020 programme under the Marie Sklodowska-Curie grant agreement No 659082.

\section{References}

[1] G. Seeman et al, Framework for Modular, Flexible and Efficient Solving the Cardiac Bidomain Equations Using PETSc, Progress in Industrial Mathematics at ECMI 2008.

[2] A. Loewe et al., Influence of the earliest right atrial activation site and its proximity to interatrial connections on P-wave morphology, EP Europace, Volume 18, Issue suppl_4, 1 December 2016.

[3] A Loewe et al, Arrhythmic potency of human ether-à-gogo-related gene mutations L532P and N588K in a computational model of human atrial myocytes, EP Europace, Volume 16, Issue 3, 1 March 2014.

[4] Bayer, J. D. et al, Novel Radiofrequency Ablation Strategies for Terminating Atrial Fibrillation in the Left Atrium : A Simulation Study, Front. Physiol. 7:108, 2016.

[5] M Valinoti et al., Phase Analysis of Endoatrial Electrograms for 3D Rotor Detection in Atrial Fibrillation, Proc. of CinC 2017.

[6] Oesterlein, T et al, Basket-Type Catheters: Diagnostic Pitfalls caused by Deformation and Limited Coverage. BioMed Research International 2016.

Address for correspondence.

Stefano Severi

DEI, University of Bologna,

Viale Risorgimento 2, 40136, Bologna, Italy

Stefano.severi@unibo.it 